\title{
NEUTRINO MASSES AND MIXINGS
}

\author{
L. Wolfenstein
}

Department of Physics

Carnegic Mellon University

Pittsburgh, PA 15213

\section{Abstract}

Theoretical prejudices, cosmology, and neutrino oscillation experiments all suggest neutrino masses are far below present direct experimental limits. Four interesting scenarios and their implications are discussed: (1) a $17 \mathrm{keV}$ $\nu_{r}$, (2) a $30 \mathrm{eV} \nu_{r}$ making up the dark matter, (3) a $10^{-3} \mathrm{eV} \nu_{\mu}$ to solve the solar neutrino problem, and (4) a three-neutrino MSW solution. 


\section{Introduction}

There is no definitive evidence that neutrinos have mass. However, the possibility that neutrinos have a non-zero mass has important implications for particle physics, cosmology, and astrophysics. In this talk I will review our theoretical ideas and experimental data on neutrino masses and mixings. Then I will focus on four particular scenarios of special interest.

Direct cvidence from decay kinematics provides the following limits:

1. From the end-point spectrum of ${ }^{3} H$ decay ${ }^{1}$,

$$
m\left(\nu_{e}\right)<9 \mathrm{eV}
$$

2. From the muon momentum in the pion decay $\pi \rightarrow \mu+\nu_{\mu}$ and an independent. measurement of the pion mass,

$$
m\left(\nu_{\mu}\right)<270 \mathrm{keV} .
$$

3. From the study of the decay $\tau \rightarrow \nu_{\tau}+5 \pi$,

$$
m\left(\nu_{\tau}\right)<35 \mathrm{MeV} \text {. }
$$

These limits are the product of a great deal of work and are probably not quite so good as indicated. (The limits on $\nu_{e}$ and $\nu_{\mu}$ come from measurements which give a central value for $m^{2}$ that is negative; the limit on $\nu_{r}$ is based on very few events.) In any case it is unlikely these limits will be significantly improved in the foreseeable future.

A very different direct way of constraining neutrino masses is the measurement of arrival times of supernova neutrinos. The data on $\bar{\nu}_{e}$ times and energies from supernova 1987 a provided a limit of about $15 \mathrm{eV}$ on the mass of the $\nu_{e}$. It is possible that if proper instrumentation is in place a large range of $\nu_{\mu}$ or $\nu_{\tau}$ mass could be explored from the study of neutrinos from the next supernova in our galaxy. ${ }^{2}$

There are a number of reasons for believing that if neutrinos have mass, the masses are far below the present limits. The reasons involve a combination of theoretical ideas, cosmology, neutrino oscillation experiments, and solar neutrino observations. These are reviewed in subsequent sections.

\section{Theoretical Ideas or Prejudices}

Massive neutrinos can either be Dirac or Majorana particles. For a Dirac neutrino the theory starts with $\nu_{L}$ as part of a weak $\mathrm{SU}(2)$ doublet and $\nu_{R}$ as a singlet, just like all the fundamental fermions. By CP or CPT $\nu_{L}$ has a righthanded antiparticle $\nu_{R}^{c}$ and $\nu_{R}$ a left-handed antiparticle $\nu_{L}^{c}$. The mass operator then connects $\nu_{L}$ to $\nu_{R}$ forming a four-component Dirac neutrino. The Majorana alternative is never to introduce $\nu_{R}$ (at least in the effective low energy theory) so that the mass term connects $\nu_{L}$ to $\nu_{R}^{c}$ thus violating lepton number by two units. The Majorana neutrino has just two components and is its own antiparticle.

Phenomenologically it is very hard to distinguish between Dirac and Majorana neutrinos, because with the usual weak interactions both transform into Weyl neutrinos in the zero mass limit (in this limit the Dirac $\nu_{R}$ decouples from the physical world). One distinction is that if one is sensitive to the right-handed component coupled by the mass, it doesn't interact for the case of a Dirac neutrino but has the normal weak interactions (of the anti-particle) for the case of a Majorana neutrino. (This will be important when we discuss the supernova later.) Another difference is that the violation of lepton number in the case of a Majorana neutrino can lead to the process of neutrinoless double beta-decay

$$
(Z, A) \rightarrow(Z+2, A)+e^{-}+e^{-} .
$$

This can be used to limit the Majorana mass of $\nu_{e}$; from the limits on ${ }^{76} \mathrm{Ge}$ double beta decay ${ }^{3}$

$$
M_{M a j}\left(\nu_{e}\right)<1 \text { to } 2 \mathrm{eV}
$$

This quantity is not a mass eigenvalue; but rather a diagonal element of the Majorana mass matrix; in terms of the mass eigenvalues $m_{i}$

$$
m_{M a j}\left(\nu_{e}\right)=\sum_{i} m_{i}\left|U_{e i}\right|^{2} \eta_{i}<1 \text { to } 2 \mathrm{eV}
$$


where $\left|U_{e i}\right|^{2}$ is the percentage admixture of $\nu_{c}$ in the state $i$ with mass $m_{i}$, and $\eta_{i}= \pm 1$ is the CP eigenvalue of state $i$.

In the standard model one can simply omit $\nu_{R}$ in the cast of characters, lepton number is conserved, and so there is no way to give the neutrinos a mass. The simplest modification is to add $\nu_{R}$ and give neutrinos a Dirac mass just as one does for all the other fermions. The problem with this is that it provides no explanation for the small values of neutrino masses; for example, $m\left(\nu_{e}\right) / m_{e} \leq 10^{-5}$.

A more attractive possibility is that neutrino masses arise from the violation of lepton number at some new high mass scale $M$. Such a lepton number violation leads to an effective (non-renormalizable) term in the low-energy SU(2) invariant weak Hamiltonian.

$$
H_{e f f}=\frac{f_{i j}}{M} \bar{\nu}_{L i} \nu_{R j}^{c} \Phi^{o} \Phi^{o}+h . c .
$$

where $\Phi^{\circ}$ is the Higgs field. Two doublets are needed bccause the transition $\nu \rightarrow \bar{\nu}$ violates weak isospin by one. When $\Phi^{o}$ gets its vacuum expectation value $v$ we obtain the Majorana mass matrix

$$
\mu_{i j}=f_{i j} v^{2} / M \text {. }
$$

This is the so-called see-saw formula because the larger the value of $M$ the smaller the resulting neutrino masses. The original version (Gell-Mann, Ramond, and Slansky) of this was in the framework of the $S O(10)$ grand unified theory. In this theory $\nu_{R}$ is required but is given a large Majorana mass $M_{R}$. The $6 \times 6$ mass matrix then takes the form

$$
\begin{array}{ccc} 
& \nu_{L} & \nu_{L}^{c} \\
\nu_{R}^{c} & 0 & M_{D} \\
\nu_{R} & M_{D} & M_{R}
\end{array}
$$

where $M_{D}$ is the usual Dirac mass matrix. One then finds the effective Majorana mass matrix for the light neutrinos is

$$
\mu \sim \frac{M_{D}^{2}}{M_{R}}
$$

In $S O(10)$ the neutrino Dirac matrix $M_{D}$ is expected to be similar to the mass matrix of the up-quarks.

A general feature of any theory of neutrino masses is the presence of mixing, analogous to that well-known for quarks. This means that the flavor states $\left(\nu_{e}, \nu_{\mu}, \nu_{\tau}\right)$ are mixtures of mass eigenstates. For example,

$$
\nu_{e}=U_{e 1} \nu_{1}+U_{e 2} \nu_{2}+U_{e 3} \nu_{3} \text {. }
$$

Again analogy with the quark sector suggests there is a mass hierarchy

$$
m\left(\nu_{3}\right) \gg m\left(\nu_{2}\right) \gg m\left(\nu_{1}\right)
$$

such that $\nu_{e}$ is mainly $\nu_{1}$, with a small admixture $\left(U_{e 2} \sim 0.2\right.$ to 0.05$)$ of $\nu_{2}$ and a still smaller admixture of $\nu_{3}$. In the case of the seasaw formula $\mathrm{Eq}$. (2) based on $\mathrm{SO}(10)$

$$
m\left(\nu_{i}\right)=m^{2}\left(u_{i}\right) / M_{i},
$$

where $m\left(u_{i}\right)$ is the mass of the up-type quark $\left(m\left(u_{3}\right)=m(\right.$ top $)$, etc). $\operatorname{SO}(10)$ doesn't tell us whether the large mass $M_{i}$ scales with the generation or is more or less constant.

\section{Cosmological Constraints}

There is a strong reason to believe that there is a background of neutrinos similar to the microwave background of photons. The same cosmological argument that explains the microwave background going back a little further in time yields the conclusion that for each type of neutrino there is a relic background of about $120 / \mathrm{cm}^{3}$. This means that the number of neutrinos is about $10^{9}$ times that of nucleons. Therefore if the neutrino mass is greater than $1 \mathrm{eV}$ or so the neutrinos provide the dominant energy density of the universe. 
The energy density of the universe determines the rate at which the expansion is slowing down. Given Hubble's constant (the present rate of expansion) and the energy density one can then calculate the "age" of the universe, that is the time since the universe was very dense. There are strong reasons to believe that the age of the universe is greater than $10^{10}$ years and this leads to the $\mathrm{imit}^{4}$

$$
\sum_{i} m\left(\nu_{i}\right) \leq 80 \mathrm{eV}
$$

provided the Hubble constant is greater than $50(\mathrm{~km} / \mathrm{sec}) / \mathrm{Mpc}$. Assuming the hierarchy of Eq. (3) this means the largest mass, essentially $m\left(\nu_{\tau}\right)$, is less than $80 \mathrm{eV}$ and the other two, $m\left(\nu_{\mu}\right)$ and $m\left(\nu_{e}\right)$, are much less than that.

There is a way out from these conclusions. If a neutrino has a mass it is possible that early in the history of the universe it decayed. The more massive the neutrino the earlier it must decay in order that its decay products (assumed to be massless) can redshift enough so as not to provide too much energy density at the present time. This leads to the constraint on the lifetime $e^{5}$

$$
\tau_{\nu}<7 \times 10^{6} \text { yrs }\left(1 \mathrm{keV} / m_{\nu}\right)^{2} .
$$

If this constraint is just barely satisfied it means that the universe is radiationdominated (this means dominated by the energy density of massless particles) from the time of decay until the present. In this case it is hard to understand how structure could have formed which has led to the suggestion of the stronger, although less rigorous, constraint ${ }^{6}$

$$
\tau_{\nu}<10^{3} y r\left(1 \mathrm{keV} / m_{\nu}\right)^{2}
$$

The only neutrino decay involving known particles would be the decay of a heavy neutrino (say $\left.\nu_{3}\right)$ to three light, neutrinos $\left(\nu_{1}\right.$ or $\left.\nu_{2}\right)$. It is hard, but not impossible, to dream up a theory to make this decay fast enough. Most theories of heavy decaying neutrinos use the decay into a light neutrino and a massless Goldstone boson, called the majoron. ${ }^{7}$ This is the Goldstone boson associated with the spontaneous violation of lepton number which can occur in models of the
Majorana neutrino mass. Another alternative in some theories is that neutrinos in the early universe annihilate into majorons

Nevertheless the simplest possibility is that Eq. (5) is satisfied. If the equality holds then the energy density of neutrinos is approximately equal to the critical density needed just to close the universe. Of particular interest is the possibility that $\Omega$ (Energy density/critical density) for neutrinos is exactly unity and massive neutrinos provide the dark matter in the universe. For a Hubble constant around $60 a \nu_{r}$ mass of around $35 \mathrm{eV}$ would be required.

\section{Neutrino Oscillations}

A consequence of neutrino masses and mixings is the phenomenon of neutrino oscillations. Considering only two generations as an example

$$
\begin{gathered}
\nu_{e}=\nu_{1} \cos \theta_{v}+\nu_{2} \sin \theta_{v} \\
\nu_{\mu}=-\nu_{1} \sin \theta_{v}+\nu_{2} \cos \theta_{v}
\end{gathered}
$$

where $\theta_{v}$ is the vacuum mixing angle. After a time $t$ a beam that was originally $\nu_{e}$ becomes

$$
\nu_{1} \cos \theta_{v} e^{-i E_{1} t}+\nu_{2} \sin \theta_{v} e^{-i E_{2} t} .
$$

Because of the mass difference between $\nu_{1}$ and $\nu_{2}$,

$$
\left(E_{2}-E_{1}\right) \approx \frac{m_{2}^{2}-m_{1}^{2}}{2 p} \equiv \frac{\Delta m^{2}}{2 p}
$$

and thus the two components get out of phase with each other. Thus the state vector is no longer purely $\nu_{e}$ but has an overlap with $\nu_{\mu}$. The probability that $\nu_{e}$ has been transformed (oscillated) into $\nu_{\mu}$ is

$$
P\left(\nu_{e} \rightarrow \nu_{\mu}\right)=\sin ^{2} 2 \theta_{v} \sin ^{2}\left(\frac{\pi x}{\ell_{v}}\right)
$$

where we replaced time by the distance traveled and

$$
\ell_{v}=4 \pi p / \Delta m^{2}
$$

is the vacuum oscillation length. 
Many experiments have been carried out searching for neutrino oscillations but no definitive evidence for oscillations has been found. A summary of limits for $\nu_{\mu}-\nu_{e}$ oscillations is shown ${ }^{8}$ in Figure 1. Most of the experiments involve the search for the appearance of $\nu_{e}\left(\bar{\nu}_{e}\right)$ from a $\nu_{\mu}\left(\bar{\nu}_{\mu}\right)$ beam. An exception is the Gosgen reactor experiment which looks for the disappearance of $\bar{\nu}_{e}$. Such disappearance experiments are limited to rather large mixing angles but provide limits on $\bar{\nu}_{e}-\bar{\nu}_{\tau}$ oscillations as well as $\bar{\nu}_{e}-\bar{\nu}_{\mu}$.

If we are guided by our theoretical prejudice that $\sin ^{2} 2 \theta_{v}$ for $\nu_{\mu}-\nu_{e}$ oscillations is of the order $10^{-2}$ or greater and that $m\left(\nu_{\mu}\right) \gg m\left(\nu_{e}\right)$ then we are led from Fig. 1 to conclude that $m\left(\nu_{\mu}\right)$ is probably less than $1 \mathrm{eV}$. Conversely, if we believe that neutrinos are stable we are led to the limit on $m\left(\nu_{r}\right)$ discussed in the cosmology section and so indeed expect that $m\left(\nu_{\mu}\right)$ is well below $1 \mathrm{eV}$. From this point of view oscillation searches simply have not gone down far enough in mass.

To probe lower values of $\Delta m^{2}$ it follows from Eq. (7) that one must look for oscillations over larger distances or at lower energies. For example, the Cal Tech collaboration $^{9}$ hopes to do reactor experiments (probably at San Onofre) to get down to $\Delta m^{2}$ of $10^{-3} \mathrm{eV}^{2}$ and eventually even lower but always at fairly large mixing angles. There is also a Los Alamos proposal to extend their range of $\Delta \mathrm{m}^{2}$. One can probe much lower values of $\Delta m^{2}\left(10^{-4}\right.$ to $\left.10^{-8} \mathrm{eV}^{2}\right)$ using neutrinos from the sun.

\section{Solar Neutrinos ${ }^{10}$}

The energy of the sun is believed to be the result of a sequence of nuclear reactions that convert hydrogen into helium. Most of this energy is produced in the form of particle energy or photons and is slowly transferred to the surface mainly by radiative transfer. However about two percent of the energy comes off in the form of neutrinos which emerge from the center with negligible absorption. The observation of these neutrinos has provided direct evidence for the processes occurring in the deep solar interior.

The major neutrino sources are shown in Table 1. The pioneering experiment of Davis detects $\nu_{e}$ from $\nu_{e}+{ }^{37} C \ell \rightarrow e^{-}+A^{37}$ using radiochemical methods. The more recent experiment of the Kaniokande group in Japan uses the reaction $\nu_{e}+e^{-} \rightarrow \nu_{e}+e^{-}$detecting the recoil electrons in a water Cerenkov detector. Since

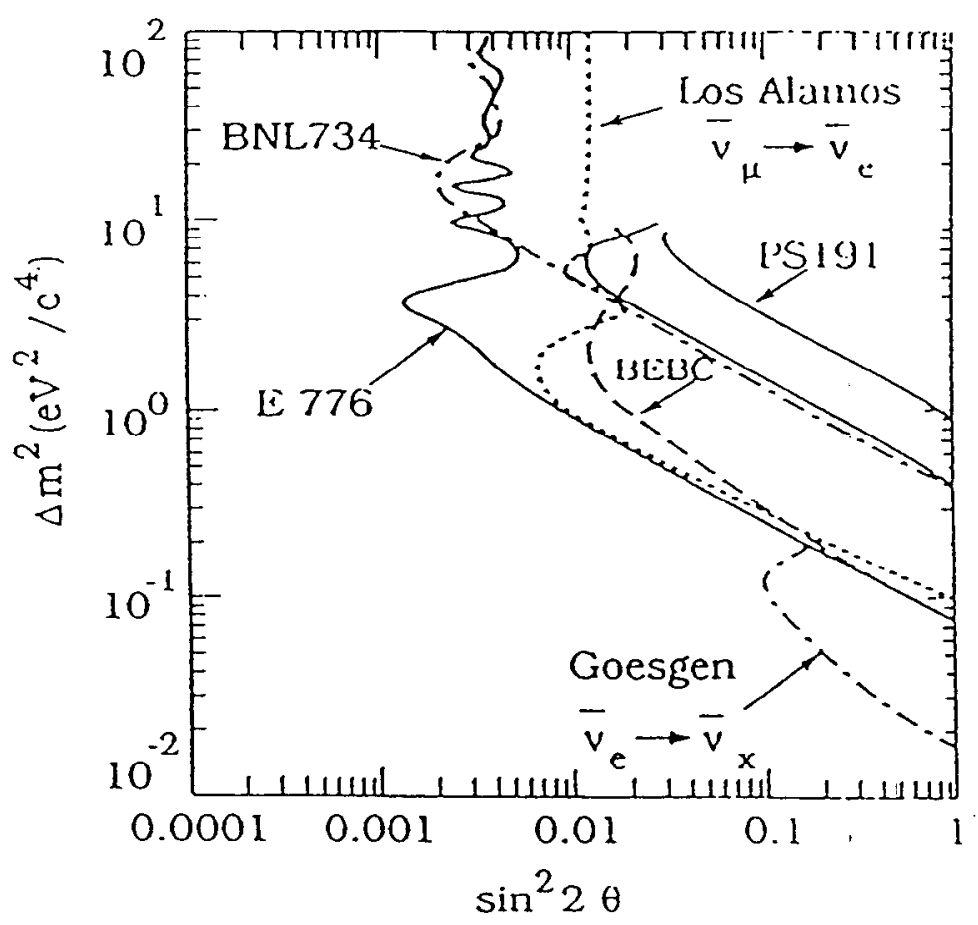

FIGURE 1. Limits on $\Delta m^{2}, \sin 2 \theta_{\mathrm{e} \mu}$ for $\nu_{\mu}-\nu_{\mathrm{c}}$ oscillations from Reference 8. 
the recoil electrons move approximately in the direction of the $\nu_{e}$ this experiment provides direct evidence that they see a signal from the sun. The Kamiokande detector is only sensitive to the rare ${ }^{8} B$ neutrinos and most of Davis' signal is also expected to come from these neutrinos.

\section{Table 1 - Major Sources of Solar Neutrinos}

\begin{tabular}{llll} 
Name & \multicolumn{1}{c}{ Reaction } & \multicolumn{1}{c}{ Energy Spectrum } & Relative Flux \\
\hline$p p \nu$ & $p+p \rightarrow d+e^{+}-\nu_{e}$ & Continuous to $420 \mathrm{keV}$ & 1 \\
${ }^{7} B e \nu$ & $e^{-}+7 B e \rightarrow^{7} L i+\nu_{e}$ & Line Mainly $860 \mathrm{keV}$ & .08 \\
${ }^{8} B \nu$ & ${ }^{8} B \rightarrow^{8} B e+e^{+}+\nu_{e}$ & Continuous to $14 \mathrm{MeV}$ & $10^{-4}$
\end{tabular}

The expected counting rates have been calculated using the standard solar model (SSM). This involves a calculation of the solar interior based upon wellestablished principles of mechanics, atomic physics, and nuclear physics. There are no conditions inside the sun that require physics beyond that studied on earth. (A possible, but very unlikely, exception would be the presence in the solar interior of a special kind of weakly-interacting particle left over from the big bang. ${ }^{11}$ )

For the Davis experiment two detailed calculations give the prediction:

$$
\begin{array}{ll}
\text { Babcall - Ulrich } & 7.9(1 \pm 0.11) \mathrm{SNU} \\
\text { Turck - Chieze }^{13} & 5.8(1 \pm .23) \mathrm{SNU}
\end{array}
$$

The theoretical uncertainties (to be considered as one standard deviation) are based entirely on estimates on the uncertainty in the input parameters (primarily nuclear cross-sections, chemical composition, and calculated opacity). The disagreement between the two calculations is primarily due to the difference in choices of these parameters. I believe a reasonably conservative value to use is $6.9(1 \pm .16) \mathrm{SNU}$ and this is used in the comparisons below. The error does not include any uncertainty due to the simplifying approximations used in the calculation; there is no reason to believe these are large but they are difficult to estimate.

The result of the Davis experiment averaged over the period from 1970 to 1990 is

$$
\text { Davis average } / \mathrm{SSM}=0.31 \pm .05 .
$$

Only the statistical error is shown; no estimate is given of systematic error, but the fluctuation of the data with time could be an indication of a non-negligible systematic error. The result of the Kamiokande experiment in the period 1986 to 1990 is

$$
\text { Kamiokande } / \mathrm{SSM}=.55 \pm .05 \pm .06
$$

where the first error is statistical and the second systematic. It is not clear whether the disagreement between the two experiments is really significant. It should be noted, however, that there are at least two reasons why the experiments might not give the same answer:

1. Kamiokande measures only ${ }^{8} B$ neutrinos whereas $25 \%$ of Davis' expected result involves lower energy neutrinos (around $1 \mathrm{MeV}$ ) such as those from ${ }^{7} B e$ and CNO reactions.

2. If one assumes that the deviation of the Davis result from the SSM is a sign of neutrino oscillations then one must take into account the fact Kamiokande is sensitive to $\nu_{\mu}$ and $\nu_{\tau}$ since they also scatter from electrons, although with a cross-section down by a factor of 6 to 7 . Thus the result 0.31 of Davis would translate into 0.41 for Kamiokande for this reason alone.

The results of these two experiments provide a hint that neutrinos oscillations may be taking place. Given the theoretical uncertainty and the difficulties of the experiment it is not yet possible to consider this as definitive evidence.

From the time Davis started his experiment it was realized that the detected flux could be affected by vacuum neutrino oscillations. Considering only $\nu_{e}$ and $\nu_{\mu}\left(\right.$ or $\nu_{e}$ and $\left.\nu_{\tau}\right)$ any value of $\Delta m^{2}$ greater than $10^{-9}(\mathrm{eV})^{2}$ would produce a reduction in the $\nu_{e}$ flux of a factor of two if the mixing were maximal. An even greater reduction could occur if there were "maximal" mixing among three neutrinos or if $\Delta m^{2}$ was $10^{-10} \mathrm{eV}^{2}$ (so that $\ell_{v}$ is tuned to the earth-sun distance).

Our theoretical prejudices do not favor such large mixing. It turns out that if $\nu_{e}$ is the lighter neutrino there exists a range of $\Delta m^{2}$ between $10^{-4}$ and $10^{-7}(\mathrm{eV})^{2}$ where large reductions of the $\nu_{e}$ flux are expected even for relatively small mixing angles. This happens as a result of neutrino oscillations that take place inside the sun as the neutrinos move from the center where they are produced to the surface. The neutrino transformation probability can be very different inside a material medium than in the vacuum. Thus even though the vacuum mixing angle $\theta_{v}$ 
is very small, the effective mixing angle in the medium can be maximal. This has been called "resonant amplication" of neutrino oscillations and is commonly referred to as the MSW (Mikhacyzev-Smirnov-Wolfenstein) effect.

The fundamental idea is that neutrinos have an index of refraction proportional to the forward elastic scattering angle. Considering only the $\nu_{e}$ scattering from electrons due to $\mathrm{W}$ exchange

$$
p(n-1)=-\sqrt{2} G N_{e}
$$

where $N_{e}$ is the number of electrons/cc and $\mathrm{G}$ is the Fermi constant. (Scattering due to neutral currents is the same for all flavors and is therefore not relevant for oscillations.) Because oscillations are a phase phenomenon the phase change due to $[p(n-1) x]$ that occurs only for $\nu_{e}$ must be included. This defines a characteristic length $\ell_{n}$ over which this phase change is $2 \pi$

$$
\ell_{o}=\left(1.6 \times 10^{7} / \rho_{e}\right) \text { meters , }
$$

where $\rho_{e}$ is the electron density in units of Avogadro's number. When $\ell_{o}$ becomes of the order of $\ell_{v}$ a large transformation of $\nu_{e}$ into $\nu_{\mu}\left(\right.$ or $\left.\nu_{r}\right)$ can take place. This occurs for

$$
p_{\nu}(\mathrm{Mev}) \rho_{\mathrm{e}}=6.5 \times 10^{6} \Delta M^{2}(\mathrm{eV})^{2}
$$

For a given $\rho_{e}$ the lower the neutrino energy the smaller the value of $\Delta m^{2}$ for the MSW effect to operate.

Many calculations of the MSW effect for the sun can be found in the literature. It is possible to get a good fit to both the Davis and Kamiokande experiments for values of $\Delta m^{2}$ from $10^{-7} \mathrm{eV}^{2}$ to $10^{-5} \mathrm{eV}^{2}$ with not very large vacuum mixing angles given by

$$
\left(\Delta m^{2}\right) \sin ^{2} 2 \theta_{v} \simeq 4 \times 10^{-8}(\mathrm{eV})^{2}
$$

While there exists considerable theoretical uncertainty concerning the flux of the ${ }^{8} B$ neutrinos, there is very little about that of the predominant pp neutrinos. This is because the pp reaction has to be the first step in fusing hydrogen in the sun so that for all reasonable calculations the flux of $\operatorname{pp} \nu$ is closely related to the total energy flux from the sun. A major advance in solar neutrino detection is now beginning with detectors capable of observing these low energy neutrinos. These are radiochemical detectors based on the inverse beta reaction

$$
\nu_{e}+{ }^{71} \mathrm{Ga} \rightarrow e^{-}+{ }^{71} \mathrm{Ge} .
$$

The SAGE (Soviet-American Gallium Experiment) began last year at the Baksan laboratory in the Caucuses. It is now taking data using 50 tons of metallic gallium. A second experiment GALLEX located in the Gran Sasso tunnel is just beginning to take data with 30 tons of gallium in the form of gallium chloride.

The calculated rates for the gallium are ${ }^{14}$

$$
\begin{array}{rcc}
p p \nu & 71 \pm 2 & S N U \\
{ }^{7} B e \nu & 34 \pm 3 & S N U \\
{ }^{8} B & (14 \pm 4) y & S N U \\
\text { Other } & (13 \pm 2) & S N U
\end{array}
$$

where $\mathrm{y}$ is a reduction factor to take into account the observed Kamiokande and Davis result that ${ }^{8} B$ neutrinos are suppressed. Taking $y=\frac{1}{2}$ the expected result within 3 standard deviations is between 110 and 140 SNU. Very preliminary results ${ }^{15}$ from SAGE based on 3 counts above background with 18 expected, give less than $72 \mathrm{SNU}$ at $90 \%$ confidence.

Figure 2 shows in the shaded portion the values of $\Delta m^{2}$ and $\sin ^{2} 2 \theta$ that give a good fit to both the average Davis and the Kamiokande data. ${ }^{16}$ Superimposed are contours showing the expected counting rate in gallium. It is clear that this range of $\Delta m^{2}$ and $\sin ^{2} 2 \theta$ allows for any result for gallium from no suppression to almost complete suppression. However, if the preliminary SAGE limit is correct and if one also demands $\sin ^{2} 2 \theta$ not too large $(<0.4)$, then one is constrained to the diagonal line (essentially given by Eq. (9)) with $\Delta m^{2}$ between $10^{-7}$ and 


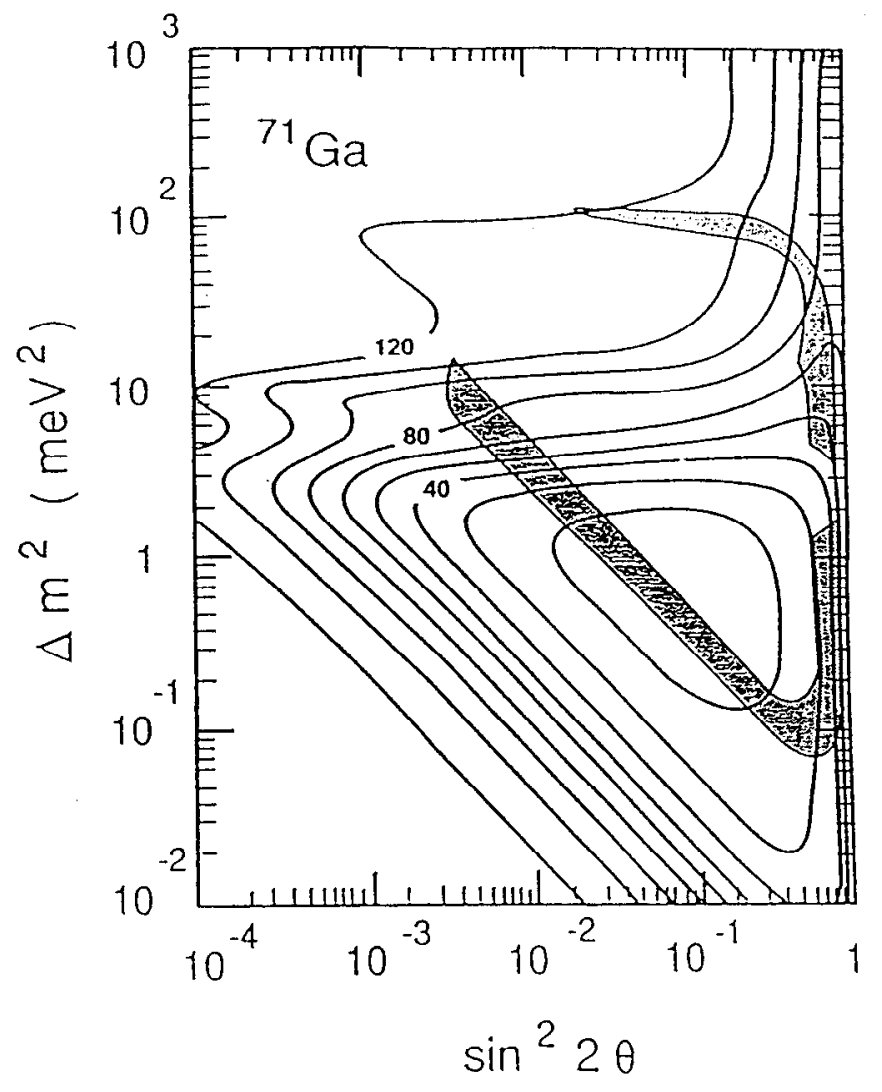

FIGURE 2. Shaded region gives values of $\Delta m^{2}$ (in units of $\left.10^{-6}(\mathrm{eV})^{2}\right)$ and $\sin ^{2} 2 \theta$ fitting Davis and Kamiokande data. Contours give SNU values for gallium experiment.
$6 \times 10^{-6}(\mathrm{eV})^{2}$. Clearly much more data is needed before any conclusions can be drawn. An alternative MSW solution is discussed below (Scenario 4).

\section{Four Scenarios}

6.1 Scenario 1 - The $17 \mathrm{keV}$ Neutrino ${ }^{17}$

There exists the interesting possibility that $\nu_{\tau}$ and/or $\nu_{\mu}$ may exceed the cosmological limit of Eq. (5) for a stable neutrino. There exists a large range of masses between the direct limits given in Sect. 1 and $80 \mathrm{eV}$ for $\nu_{\mu}$ and $\nu_{r}$. These can only be explored by experiments depending on neutrino mixing or possibly a future supernova. As an illustration we concentrate on a $17 \mathrm{keV} \nu_{\tau}$ with a mixing with $\nu_{e}$ given by $\left|U_{e 3}\right|^{2}=.01$. As discussed in the previous talk evidence relevant to such a neutrino can be deduced from a study of beta spectra. (Note from Figure 1 that $1 \%$ mixing between $\nu_{e}$ and $\nu_{\mu}$ is ruled out by oscillation experiments.)

The simplest assumption is that this $17 \mathrm{keV} \nu_{r}$ is a Dirac neutrino. There is then no trouble with double beta decay and one can even imagine masses for $\nu_{\mu}$ and $\nu_{e}$ that could solve the solar neutrino problem. However, for reasons discussed below, we may prefer the Majorana solution. Then there appears to be a problem with the double beta-decay constraint of Eq. (1) since

$$
m_{3}\left|U_{e 3}\right|^{2}=170 \mathrm{eV} \gg 1 \text { to } 2 \mathrm{eV} \text {. }
$$

Thus it is necessary that the other terms in Eq. (1) cancel this term. Such a cancellation is not necessarily unnatural since the quantity $M_{M a j}\left(\nu_{e}\right)$ being constrained, a diagonal element of the mass matrix, may vanish in some models as a result of a symmetry condition. The simplest way to achieve this is a model in which the lepton number combination $L_{e}+L_{r}-L_{\mu}$ is conserved even though the total lepton number is not. This conservation law forbids double beta-decay. The Majorana mass matrix then takes the form

$$
\begin{array}{lll} 
& \left(\nu_{\mu}\right)_{L} & \left(v_{\tau}+0.1 \nu_{e}\right)_{L} \\
\left(\nu_{\mu}\right)_{R}^{c} & 0 & \mathrm{~m} \\
\left(\nu_{\tau}+0.1 \nu_{e}\right)_{R}^{c} & \mathrm{~m} & 0
\end{array}
$$

There are then two $17 \mathrm{keV}$ Majorana states with opposite values of $\eta$ given by 


$$
\left(\nu_{\tau}+0.1 \nu_{e}\right) \pm \nu_{\mu}
$$

Thus $\nu_{\mu}$ also has a mass of $17 \mathrm{keV}$. In fact since there is a conserved lepton number we have a kind of Dirac neutrino; indeed the mass matrix is identical to that of a Dirac neutrino except that $\left(\nu_{\mu}\right)_{R}^{c}$ (the anti-particle of $\nu_{\mu L}$ ) plays the role of the right-handed neutrino. The two degenerate Majoranas can be combined to a fourcomponent object referred to as a Zedovich-Konopinski-Mahmoud (ZKM) type of Dirac neutrino. This type of Dirac neutrino is obviously distinguished from a "normal" Dirac neutrino (the kind we discussed in Sec. 2) by the way its "righthanded component" interacts. There are also interesting different possibilities concerning the magnetic moment. ${ }^{18}$

A number of papers refer to a "pseudo-Dirac" neutrino. I first introduced this term ${ }^{19}$ to describe a modification of the ZKM scenario in which the mass matrix contained a conserved lepton number but that number was not a lepton number conserved by the weak interactions. (Such a mass matrix can be obtained from the form (10) by "rotating" the matrix.) As a result diagonalizing the mass matrix one obtained two degenerate Majoranas just as in the ZKM but these are then split by weak radiative corrections. The term "pseudo-Dirac" has been used more generally to refer to the case of any theory which contains a small parameter $\lambda$ such that in the limit $\lambda$ goes to zero two Majoranas merge into a ZKM Dirac-type neutrino.

As discussed in Sect. 3 any model of a $17 \mathrm{keV}$ neutrino must meet the cosmological constraint that the neutrino decay with the lifetime limit of Eqs. (6a) or (6b) or annihilate in the early universe. Nearly all models require the existence of the majoron and thus the violation of lepton number. This is a strong reason for expecting the $17 \mathrm{keV}$ to be a Majorana neutrino rather than a "normal" Dirac neutrino.

There is still another reason having to do with the observation of neutrinos from the supernova SN 1987a. In the hot dense core of the collapsing star large numbers of neutrinos are produced. These neutrinos interact many times because of the great density before they can emerge. However if we have a "normal" Dirac neutrino each weak interaction of a $\nu_{L}$ will yield a $\nu_{R}$ with the probability $\left(m_{\nu} / E\right)^{2}$. (This is because of the difference between chirality and helicity.) But $\nu_{R}$ interacts very weakly and so can quickly escape without any further interaction. Thus the supernova loses energy more rapidly than in the standard supernova model. As a result the pulse of $\bar{\nu}_{e}$ observed would have a shorter time duration. The observations can then be used ${ }^{20}$ to put a limit on $m_{\nu}$ for a "normal" Dirac neutrino. Calculations of this limit are still going on but a reasonable result ${ }^{21}$ seems to be $m_{\nu}<10 \mathrm{keV}$, which marginally rules out a $17-\mathrm{keV}$ Dirac neutrino. Of course, this does not rule out a $17-\mathrm{keV}$ ZKM neutrino.

\subsection{Scenario 2 - Neutrinos as Dark Matter}

A fascinating possibility is that the heaviest neutrino, persumably $\nu_{\tau}$, just meets the cosmological bound for a stable neutrino and indeed provides most or all of the dark matter of the universe. In particular for a Hubble constant of about 60 a mass of $\left(\nu_{\tau}\right)$ of $30 \mathrm{eV}$ would result in $\Omega_{\nu}=1$; that is, the massive neutrinos left over provide just the critical density to close the universe.

Unfortunately it is very hard to test this possibility experimentally. Direct detection of the background $30 \mathrm{eV}$ neutrinos seems impossible. The only way I know to find some evidence that $m\left(\nu_{\tau}\right)$ is $30 \mathrm{eV}$ is to search for $\nu_{\mu}-\nu_{\tau}$ oscillations. Assuming $m\left(\nu_{\mu}\right) \ll m\left(\nu_{r}\right)$ this means searching in the region $\Delta m^{2} \sim 10^{3}(\mathrm{eV})^{2}$. Such a search was done many years ago at Fermilab (E-531) using emulsions to detect $\nu_{r} ;$ for $\Delta m^{2}>30 \mathrm{eV}^{2}$ this provided a limit on the mixing $\sin ^{2} 2 \theta_{\mu \tau}<$ $4 \times 10^{-3}$. There exists a proposal (P-803) at Fermilab again using emulsions to push the limit to $10^{-4}$ with the potential of seeing a significant signal if $\sin ^{2} 2 \theta_{\mu \tau}>$ $4 \times 10^{-4}$. There are also two related proposals at CERN, one using emulsions and one using scintillating fibers.

It should be noted, of course, that a positive signal could have a variety of interpretations. The experiment would only define a lower limit on $\Delta m^{2}$; thus the same signal could be interpreted as a $30 \mathrm{eV}$ or a $17 \mathrm{keV} \nu_{r}$. In fact because the $\nu_{\mu}$ beam contains some $\nu_{e}$ any signal could also be due to $\nu_{e}-\nu_{\tau}$ oscillations. Indeed the proposal P-803 would be capable of placing a limit of $6 \times 10^{-3}$ on $\sin ^{2} 2 \theta_{e r}$ for $\Delta m^{2}>50 \mathrm{eV}^{2}$. If there were a $17 \mathrm{keV} \nu_{\tau}$ with a mixing probability of $1 \%$ with $\nu_{e}$ then $\sin ^{2} 2 \theta_{e r}$ would be $4 \times 10^{-2}$ and so should give a signal in P-803. In spite of these ambiguities I believe this is a very important experiment. 


\subsection{Scenario 3 - MSW via $\nu_{\mu}$}

If we accept the Davis and Kamiokande experiments as requiring neutrino oscillations and if further we accept the preliminary data from the SAGE experiment, then the best fit to all the data is given by Eq. (9) with

$$
\begin{aligned}
m\left(\nu_{\mu}\right) & \sim 10^{-3} \mathrm{eV} \\
\sin ^{2} 2 \theta_{e \mu} & \sim 0.2 \text { to } 10^{-2}
\end{aligned}
$$

We have used our theoretical prejudice to limit the range of mixing angles. Of course as far as solar neutrinos are concerned we could replace $\nu_{\mu}$ by $\nu_{r}$, but we prefer $\nu_{\mu}$ for this range of mixing angles, since we expect $\nu_{e}-\nu_{\tau}$ mixing to be small.

An interesting point about this solution is the implication for the mass of $\nu_{\tau}$. According to our assumptions of a mass hierarchy a range for $\nu_{r}$ might be

$$
m\left(\nu_{\tau}\right)=0.1 \text { to } 1 \mathrm{eV} .
$$

(A more extreme mass ratio could give $30 \mathrm{eV}$, thus merging with Scenario 2.) The best hope for exploring such a mass range is to search for $\nu_{\mu}-\nu_{\tau}$ mixing. At present the only accelerator data provides limits on $\nu_{\mu}$ disappearance from the CDHSW experiment which rules out $\Delta m^{2}>0.5 \mathrm{eV}^{2}$ for $\sin ^{2} 2 \theta_{\mu \tau}>0.2$. In order to really explore the range of $\Delta m^{2}$ down to $10^{-2} \mathrm{eV}^{2}$ one clearly needs a long baseline experiment. One method is the use of cosmic-ray beams. Indeed there is evidence from the Kamiokande experiment (confirmed by IMB but not by Frejus) for a deviation of the $\nu_{\mu} / \nu_{e}$ ratio from that expected for the cosmic-ray beam. This could be explained by $\nu_{\mu}-\nu_{\tau}$ oscillations with a value of $\Delta m^{2}$ around $10^{-2} \mathrm{eV}^{2}$ and a large value of $\theta_{\mu \tau}$

However, it is difficult to be convinced by cosmic-ray data. Thus there is the hope of doing long baseline experiments with accelerator $\nu_{\mu}$ beams. One proposal at Fermilab (P-822) calls for a beam directed at the Soudan detector in Minnesota at a distance of $800 \mathrm{~km}$. In the proposal it is claimed that values of $\Delta \mathrm{m}^{2}$ down to $10^{-2} \mathrm{eV}^{2}$ could be explored for $\sin ^{2} 2 \theta_{\mu \tau}$ greater than 0.1 . The completed Souden II detector will still be quite small (1100 tons) and one would expect that if the proposal is approved the detection system would be expanded for this purpose. Notice, unlike the emulsion experiment P-803 discussed above, the detector could not identify $\nu_{\tau}$ but only the disappearance of $\nu_{\mu}$ or possibly a change in the "neutral current/charged current" ratio with most $\nu_{\tau}$ interactions counting as "neutral current." Thus the experiment is limited to relatively large mixing angles but probably would be more reliable than the cosmic-ray experiments

6.4 Scenario 4 - MSW via $\nu_{\tau}$ and $\nu_{\mu}$

A slightly bizarre possibility, but one that fits our theoretical ideas, has both $\nu_{\mu}$ and $\nu_{\tau}$ affecting solar neutrinos. We assume

$$
\begin{aligned}
m\left(\nu_{\tau}\right) & \sim 10^{-2} \mathrm{eV} \\
\sin ^{2} 2 \theta_{e \tau} & \sim 10^{-3} \mathrm{eV} .
\end{aligned}
$$

With this range of values we can fit either the Davis or Kamiokande experiments. If we insist on fitting them both we do not get a very good fit; if we fit Kamiokande, the result for Davis is somewhat too large. Because of the large value of $\Delta m^{2}$ there is no suppression of the pp neutrinos or Be neutrinos that dominate the signal for the gallium detector. Thus this $\nu_{\tau}$ cannot explain the preliminary SAGE result.

However our hierarchy assumption allows us to assume simultaneously

$$
\begin{aligned}
m\left(\nu_{\mu}\right) & \sim 10^{-4} \mathrm{eV} \\
\sin ^{2} 2 \theta_{e \mu} & \sim 10^{-1} .
\end{aligned}
$$

Thus $\nu_{e}-\nu_{\mu}$ oscillations can lead to a large supression of the pp neutrinos and the Be neutrinos and thus explain the SAGE result. ${ }^{22}$ The suppression of the Be neutrinos would also help reduce the Davis result. A proposed experiment (BOREX) focusing on the detection of the Be neutrinos might help distinguish Scenarios 3 and 4 .

The last three scenarios are all consistent with the general seesaw formula of Eq. (4). In particular, if we set $m\left(u_{3}\right)=m_{\text {top }}$ then we have for 


$$
\begin{array}{lll}
\text { Scenario 2: } & M_{3} \sim 10^{11}-10^{12} \mathrm{GeV} \\
\text { Scenario 3: } & M_{3} \sim 10^{13}-10^{14} \mathrm{GeV} \\
\text { Scenario 4: } & M_{3} \sim 10^{15} \mathrm{GeV} .
\end{array}
$$

To conclude there is still no decisive reason, theoretical or experimental, to believe in a non-zero neutrino mass. However there are many exciting scenarios that deserve further exploration.

This work was supported in part by the Department of Energy Contract No. DE-AC02-76ER03066.

\section{REFERENCES}

(For more details and more references sce my 1988 SLAC lectures or P.B Pal and R. N. Mohapatra, (World Scientific). Massive Neutrinos, Physics and Astrophysics.)

1. R.G.H. Robertson et al., Phys. Rev. Lett. 67, 957 (1991).

2. D. Cline et al., UCLA preprint APH 0029 (1990); A. Acker et al., Univ. of Hawaii preprint UH-511-688-89 (1989); J. M. Soares and L. Wolfenstein, Phys. Rev. D40, 3660 (1989); L. Krauss et al., Yale preprint YCTP P29-91 (1991).

3. D. Caldwell et al., Nuc. Phys. (Suppl) B13, 547 (1990).

4. For a useful discussion see J. Bernstein and G. Feinberg, Phys. Lett. 101B, 39 (1981).

5. D. Dicus et al., AP. J. 221, 327 (1978); P. B. Pal, Nucl. Phys. B227, 237 (1983).

6. G. Steigman and M. Turner, Nuc. Phys. B253, 375 (1985).

7. Y. Chikashige, R. Mohapatra, and R. Peccei, Phys. Lett. 98B, 265 (1981).

8. L. Moscoso, Review Talk at Neutrino 90, CERN, June 1990.

9. F. Boehm, Talk at Neutrino Workshop, L'Aquila, Italy, July 1991.

10. For more details see Davis, Mann, and Wolfenstein, Ann. Rev. Nuc. Sci. 39,467 (1989) and J. N. Bahcall, Neutrino Astrophysics, Cambridge U. Press (1989).

11. W. H. Press and D. N. Spergel, Ap. J. 296, 679 (1985); J. Faulkner and R. L. Gilliland, Ap. J. 299, 994 (1985).
12. J. N. Bachall and R. K. Ulrich, Rev. Mod. Phys. 60, 297 (1988). 1 SNU corresponds to one neutrino capture per sec per $10^{36}$ atoms.

13. S. Turck-Chieze et al., Ap. J. 335, 415 (1988).

14. These results are from Ref. 12 with one standard deviation errors indicated Uncertainties due to the flux calculations and those due to the gallium crosssection have been added linearly. Very similar results with smaller estimated errors are given by $\mathrm{W}$. Hampel. Int Symp. on Weak and Electromagnetic Interactions in Nuclei, (ed. H. V. Klapdor) p. 718, Springer-Verlag (1986).

15. J. Wilkerson, Talk at Neutrino Workshop, L'Aquila, Italy, July 1991.

16. This is taken from the work of S. Bludman et al., University of Pennsylvania UPR 09437 (April 1991). Very similar fits to the data have been given in many papers. See, for example, V. Barger et al., Phys. Rev. D43, 1110 (1991).

17. Countless theoretical papers discuss the $17 \mathrm{keV}$ neutrino. For a good review and many references see $G$ Gelmini, S. Nussinov, and R. Peccei, UCLA preprint UCLA/91/TEP/15 (1991).

18. L. Wolfenstein, Nuc. Phys. B345, 327 (1990).

19. L. Wolfenstein, Nuc. Phys. B186, 147 (1981).

20. G. Raffaelt and D. Seckel, Phys. Rev. Lett. 60, 1793 (1988).

21. See M. Turner, Univ. of Chicago preprint elaborating on the work of $A$. Burrows and R. Gandhi, Phys. Lett. 246B, 149 (1990).

22. This is one of the possibilities shown in Table 1 of my Sendai Talk, Neutrino 86 (eds. T. Kitagaki and H. Yuta), p. 12, World Scientific (1986). 